\section{Cerebritis bifrontal y absceso cerebral por Streptococcus del grupo anginosus, en paciente previamente sano. Caso clínico}

\author{
JOSÉ MANUEL MATAMALA ${ }^{1}$, CAROLINA NÚÑEZ ${ }^{1}$, \\ ROSA OGRODNIK $^{1, a}$, LUIS CARTIER ${ }^{1,2}$
}

\section{Bifrontal cerebritis and brain abscess caused by Sreptococcus anginosus group. Report of one case}

The etiology of brain abscesses is mostly polymicrobial. Streptococci and anaerobic bacteria are the most commonly isolated pathogens. We report a previously healthy female without predisposing factors, presenting with a bifrontal cerebritis caused by a Streptococcus anginosus group infection. The patient developed a brain abscess and a subdural collection with severe intracranial hypertension of fatal evolution. The etiologic diagnosis was made culturing the material obtained from the subdural collection. It is presumed that, within the Streptococcus anginosus group, Streptococus intermedius could have been the causing bacteria, given its central nervous system tissue tropism and its predisposition to form brain abscesses.

(Rev Med Chile 2013; 141: 109-113).

Key words: Brain abscess; Brain diseases; Streptococcus anginosus.
'Departamento de Ciencias Neurológicas Oriente, Facultad de Medicina, Universidad de Chile. ${ }^{2}$ Servicio de Neurología, Hospital del Salvador. aAlumno de Medicina, Facultad de Medicina, Universidad de Chile.

Financiamiento: Sin financiamiento.

Conflicto de interés: Los autores declaran no presentar conflicto de intereses.

Recibido el 20 de abril de 2012, aceptado el 31 de agosto de 2012

Correspondencia a: Dr. Luis Cartier Rovirosa. Avenida Salvador 364,

Providencia.

Teléfono: +56-2-5754000; E-mail: Icartier@med. uchile.cl.
L a cerebritis define la inflamación difusa de un sector del tejido nervioso, que puede evolucionar hacia la necrosis, dando origen a un absceso cerebral, que configura una colección purulenta delimitada por una cápsula glial densa, ricamente vascularizada ${ }^{1}$. Hasta el siglo XIX los abscesos cerebrales eran invariablemente fatales. La neurocirugía primero y la era antibiótica después, asociados ahora a la tomografía computada y la resonancia magnética han permitido reducir la morbimortalidad, especialmente debido a la precocidad diagnóstica ${ }^{2}$. Estas lesiones que se expresan con mayor frecuencia entre los 20 y 40 años, afectan preferentemente a varones (relación 1.3:1 a 3.0:1 $)^{2,3}$. Las infecciones rinosinusales, óticas, traumatismos encefalocraneanos y procedimientos neuroquirúrgicos suelen ser los factores inductores. Otros contribuyentes son la diabetes mellitus, la insuficiencia hepática o renal y las cardiopatías congénitas ${ }^{1}$. El 20 a 30\% de abscesos cerebrales no presentan antecedentes desencadenantes ni predisponentes ${ }^{1,4}$.
Los abscesos cerebrales se sospechan clínicamente por una triada sintomática de cefalea, fiebre y signos focales, que sólo un tercio de los pacientes completa. La cefalea es el síntoma más significativo por su intensidad y persistencia, la fiebre aparece solamente en la mitad de los casos. La signología meníngea, el edema de papila o las crisis convulsivas están presentes en $25 \%{ }^{5,6}$. El desarrollo de meningitis es frecuente, sin embargo, la mayoría de los abscesos son solitarios y tienen preferencia por el lóbulo frontal ${ }^{5,6}$. Los déficits focales se relacionan con la ubicación, el tamaño y el número de lesiones ${ }^{1,6}$.

Los agentes que se aíslan incluyen a los Streptococcus y bacterias anaerobias ${ }^{7}$. Los Streptococcus del grupo anginosus (SAG), son reconocidos como los agentes patógenos más frecuentes en los abscesos cerebrales. Los SAG son cocáceas grampositivas, en cadena, catalasa negativa y forman parte del grupo del Streptococcus viridans. Pueden carecer del carácter hemolítico, o generar hemólisis tipo $\beta$ ó $\alpha^{8,9}$. Son organismos habitualmente comensales 
saprófitos que circunstancialmente se tornan patógenos. Dentro del grupo anginosus se conocen tres especies: Streptococcus anginosus, Streptococcus constellatus y Streptococcus intermedius, siendo este último, el más habitual en la génesis de cerebritis $^{10,11}$.

Se presenta el caso de una paciente con una cerebritis bifrontal por SAG que desarrolló un absceso cerebral y una severa hipertensión endocraneana de evolución fatal.

\section{Caso clínico}

Mujer de 46 años hipertensa controlada, que presentó cefalea holocránea acompañada de náuseas, vómitos y fiebre, cuya intensidad llegó a 10/10 al tercer día de evolución. La aparición de compromiso de conciencia obligó a consultar en Servicio de Urgencias. Ingresó confusa, lenguaje poco fluente, rigidez de nuca, pupilas isocoricas reactivas y fondo de ojo con edema de papila bilateral. Movilizaba las 4 extremidades, tenía reflejos osteotendíneos vivos y Babinski bilateral. Presión arterial 108/64 $\mathrm{mmHg}$, frecuencia cardiaca 56 $\operatorname{lmp}$, subfebril $\left(\mathrm{t}^{\circ} 37,2^{\circ} \mathrm{C}\right)$. Sin lesiones en mucosas o piel y ausencia de focos dentarios, sinusales, oticos o respiratorio. La tomografía cerebral (TC) mostró hipodensidad frontal izquierda redondeada, con efecto de masa local sobre estructuras adyacentes. Líquido cefalorraquídeo citrino, 760 leucocitos $\mathrm{mm}^{3}$ (97\% PMN), proteínas 101,8 mg/ $\mathrm{dL}$, glucosa $69 \mathrm{mg} / \mathrm{dL}$ (hemoglucotest de $116 \mathrm{mg} /$ dL), la tinción Gram fue negativa. Hematocrito $29,9 \%$, hemoglobina $9,8 \mathrm{~g} / \mathrm{L}$, leucocitos $25.800 \mathrm{k} /$ uL, (91,9\% segmentados), plaquetas $286.000 \mathrm{uL}$, PCR $428 \mathrm{mg} / \mathrm{L}$, protrombina 66\%, y electrolitos plasmáticos normales. VHB, VHC, HTLV-1, Chagas y VIH negativos.

Se inició tratamiento antibiótico endovenoso con ceftriaxona $2 \mathrm{~g} \mathrm{c} / 12 \mathrm{~h}$, clindamicina $600 \mathrm{mg}$ $\mathrm{c} / 8 \mathrm{~h}$, vancomicina $1 \mathrm{~g} \mathrm{c} / 12 \mathrm{~h}$ y betametasona 4 $\mathrm{mg} \mathrm{c} / 8 \mathrm{~h}$. La paciente persistió comprometida de conciencia (Glasgow 12), con rigidez de nuca y opistótono. Pupilas isocóricas reactivas, reflejos oculocefálicos y corneales conservados, mantenía el edema de papila, la hiperreflexia generalizada y Babinski bilateral. La resonancia magnética (RM), realizada al segundo día de hospitalización, definió un proceso bifrontal paramediano anterior, de predominio izquierdo, con difusión restringida y edema vasogénico perilesional. Se observó captación de contraste leptomeníngeo y se identificó una colección subdural fronto-temporal izquierda de $6 \mathrm{~mm}$. La angioresonancia no agregó elementos significativos. El estudio se interpretó como cerebritis bifrontal abscedada, empiema frontoparietal izquierdo y leptomeningitis (Figura 1). La evaluación neuroquirúrgica realizada en dicho momento sugirió manejo medico del cuadro. El eco-cardiograma y ecografía abdomino-pélvica fueron normales. Los hemocultivos, urocultivos, cultivo de secreción bronquial y cultivo de LCR fueron negativos.

Al cuarto día de hospitalización se acentuó el compromiso de conciencia (Glasgow 4). TC cerebral mostró significativo aumento del edema cerebral sin cambios en las lesiones descritas. La reevaluación neuroquirúrgica decidió evacuar la colección subdural izquierda, dando salida a material purulento. El procedimiento se desarrolló sin incidencias. En la etapa inmediata postquirúrgica necesitó apoyo con drogas vasoactivas, no recuperó la conciencia, quedando en coma, con pupilas midriáticas arreflécticas, sin respuestas de tronco. La TC mostró colapso ventricular, ausencia de surcos y borramiento de las cisternas basales. Conectada a ventilador, el electroencefalograma mostró trazado plano. Se definió muerte cerebral.

El cultivo de la colección subdural fue positivo para Streptococcus del grupo anginosus (SAG).

\section{Discusión}

Se ha presentado una paciente que desarrolló cerebritis y absceso por SAG sin antecedentes infecciosos previos identificables. En relación a la microbiología de los abscesos cerebrales, De Louvois y cols, reportava al Streptococcus spp como el organismo infectante más común (74\%), seguido por los Bacteroides spp (24\%), el Staphylococcus aureus (20\%) y el Proteus spp $(15 \%)^{12}$. Por su parte, en el Reino Unido, un estudio de 153 muestras de SAG definidas en diferentes laboratorios y en concordancia con varios autores, identificó al Streptococcus intermedius como poseedor de una marcada preferencia por las infecciones del SNC. El Streptococcus anginosus se lo relacionó con infecciones del tracto gastrointestinal y genitourinario, sin comprometer el SNC. El Streptococcus constellatus no mostró preferencia por ningún tejido, 


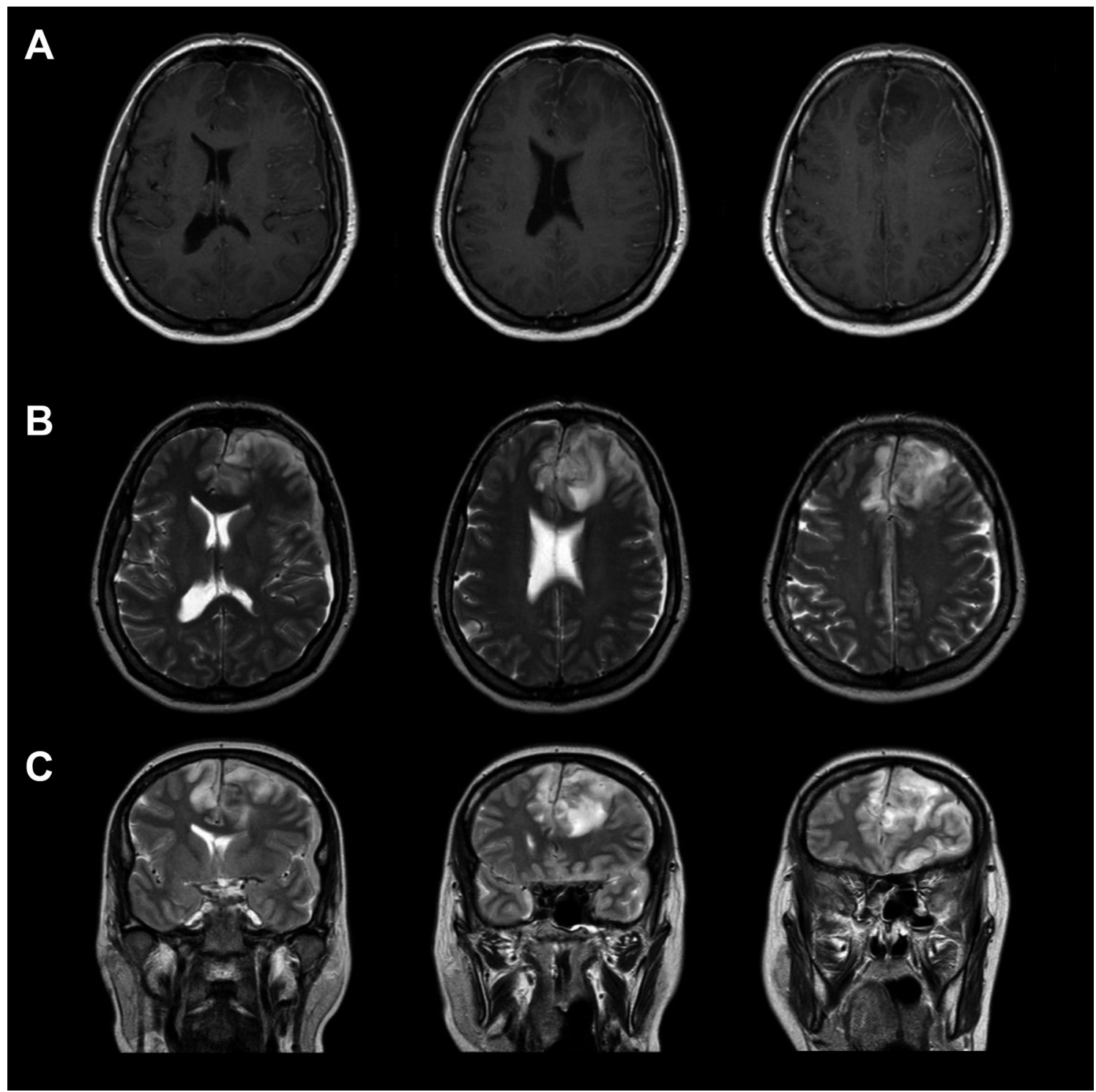

Figura 1. RM cerebral de cerebritis bifrontal y absceso cerebral secundario a Streptococcus del grupo anginosus. A. Cortes axiales en secuencia T1 con gadolinio muestra hipointesidad bifrontal mayor a izquierda con captación de contraste leptomeningeo y efecto de masa. B, C. Cortes axiales y coronales en secuencia T2 muestra hiperintensidad en región bifrontal mayor a izquierda.

infectando excepcionalmente el SNC ${ }^{13}$.

Entre los factores que podrían favorecer la virulencia de estas bacterias, habitualmente saprófitas, promoviendo su colonización a diferentes tejidos, se ha descrito una adhesina no fibrilar que desarrolla uniones con la fibronectina de las células huésped, provocando simultáneamente una relativa resistencia a la activación de los PMN e inhibiendo parcialmente las quimiotaxinas de células inflamatorias. Esta condición sería clave para permitir la colonización y posterior desarrollo de estas infecciones piógenas ${ }^{14}$. La facilidad del Streptococcus intermedius para generar abscesos se debe a la expresión de proteasas y glicosidasas (sialidasas y hialorunidasas), capaces de alterar los tejidos. Además secreta una citotoxina $(54-\mathrm{kDa})$, llamada intermedilisina (ILY), capaz de originar poros en la membrana plasmática ${ }^{15}$. Nagamune y 
cols encontraron que la cantidad de ILY producida por cepas vinculadas a infecciones profundas (abscesos cerebrales o hepáticos) era 6 a 10 veces mayor que el de las cepas encontradas en placas dentales, sugiriendo que la mayor síntesis de ILY sería la clave para la necrosis y la génesis de abscesos ${ }^{16}$. Además se ha destacado la activación del sistema de "quorum sensing" en cepas de Streptopcocus intermedius (sistema AI-2/LuxS) y su rol clave en la formación del biofilms bacterianos así como la susceptibilidad a antimicrobianos ${ }^{17,18}$. Además la activación de este sistema involucra la regulación en la expresión de factores de virulencia, como ILY $^{19}$.

La identificación de estas bacterias no siempre es sencilla, debido a su diversa reactividad, dependientes del medio de cultivo, así como del modo y tiempo de incubación ${ }^{8,10}$. Por ello la identificación y clasificación de los SAG basadas en su capacidad hemolítica y la presencia del antígeno de Lancefield resulta poco discriminatoria. Es la reacción en cadena de la polimerasa (PCR) el método que permite establecer fenotipos de manera más precisa y ha permitido identificar genes discriminatorios entre subtipos de SAG. Woo y cols aplicaron la secuencia $16 \mathrm{~S}$ del rRNA bacteriano a 6 casos de endocarditis por SAG, logrando la identificación de la especie Streptococcus anginosus como agente etiológico ${ }^{20}$. La aplicación de la amplificación y secuencia del $16 \mathrm{~S}$ rRNA bacteriano ha servido como herramienta complementaria al cultivo, proporcionando la etiología y la posibilidad de un tratamiento específico en los abscesos intracerebrales ${ }^{21}$.

En cuanto al tratamiento antimicrobiano, hasta el año 1994 no se había reportado resistencia del SAG a la penicilina ${ }^{22}$. Estudios posteriores, en el año 1996, habrían mostrado una disminución de la sensibilidad a la penicilina, macrólidos, ciprofloxacino y clindamicina ${ }^{23,24}$. Por otra parte, se ha reportado una alta sensibilidad (97-98\%) a las cefalosporinas endovenosas de tercera generación como la cefotaxima ${ }^{25}$. Así como la vancomicina, imipenem y teicoplanina muestran 100\% de sensibilidad en las infecciones por SAG intracerebrales $^{24}$. La gran mayoría de los SAG serían sensibles a dosis usuales de penicilina, amoxicilina, cefotaxima y ceftriaxona ${ }^{25,26}$.

Nuestra paciente es un buen ejemplo de la imprevisible conducta de los SAG. El diagnóstico etiológico se determinó mediante el estudio del material purulento de la colección subdural y aunque no se pudo identificar la especie de SAG mediante la reacción de cadena de la polimerasa (PCR), hay suficiente información en la literatura para presumir como causante al Streptococcus intermedius.

En conclusión, los SAG corresponden a un grupo de agentes microbianos saprofitos, capaces de generar infecciones severas del SNC en ausencia de condiciones predisponentes o focos infecciosos a distancia. Su identificación oportuna habitualmente se logra a través del estudio de material del absceso, porque usualmente los cultivos de LCR y hemocultivos son negativos. La presentación de este caso apunta a resaltar la gravedad que tienen las infecciones por SAG y que frente a un absceso cerebral se debe pensar en una infección por este agente lo que permitirá optimizar el tratamiento de manera rápida y apropiada, incluyendo el drenaje quirúrgico oportuno del absceso.

\section{Referencias}

1. Mathisen GE, Johnson JP. Brain abscess. Clin Infect Dis 1997; 25: 763-79.

2. Carpenter J, Stapleton S, Holliman R. Retrospective analysis of 49 cases of brain abscess and review of the literature. Eur J ClinMicrobiol Infect Dis 2007; 26: 1-11.

3. Menon S, Bharadwaj R, Chowdhary A, Kaundinya DV, Palande DA. Current epidemiology of intracranial abscesses: a prospective 5 year study. J Med Microbiol 2008; 57: 1259-68.

4. Roche M, Humphreys H, Smyth E, Phillips J, Cunney $\mathrm{R}, \mathrm{McNamara} \mathrm{E}$, et al. A twelve-year review of central nervous system bacterial abscesses; presentation and aetiology. Clin Microbiol Infect 2003; 9: 803-9.

5. Manzar N, Manzar B, Kumar R, Bari ME. The study of etiologic and demographic characteristics of intracranial brain abscess: a consecutive case series study from Pakistan. World Neurosurg 2011; 76: 195-200.

6. Kao PT, Tseng HK, Liu CP, Su SC, Lee CM. Brain abscess: clinical analysis of 53 cases. J MicrobiolImmunol Infect 2003; 36: 129-36.

7. Gortvai P, De Louvois J, Hurley R. The bacteriology and chemotherapy of acute pyogenic brain abscess. $\mathrm{Br} \mathrm{J}$ Neurosurg 1987; 1: 189-203.

8. Ruoff KL. Streptococcus anginosus ("Streptococcus milleri"): the unrecognized pathogen. ClinMicrobiol Rev 1988; 1: 102-8.

9. Petti CA, Simmon KE, Bender J, Blaschke A, Webster 
KA, Conneely MF, et al. Culture-Negative intracerebral abscesses in children and adolescents from Streptococcus anginosus group infection: a case series. Clin Infect Dis 2008; 46: 1578-80.

10. Palavecino E. Streptococcus grupo anginosus: ¿Es su identificación clínicamente importante? Rev Chil Infect 2004; 21: 261-67.

11. Claridge JE 3rd, Attorri S, Musher DM, Hebert J, Dunbar S. Streptococcus intermedius, Streptococcus constellatus, and Streptococcus anginosus ("Streptococcus milleri group") are of different clinical importance and are not equally associated with abscess. Clin Infect Dis 2001; 32: 1511-5.

12. De Louvois J, Gortavai P, Hurley R. Bacteriology of abscesses of the central nervous system: a multicentre prospective study. Br Med J 1977; 2: 981-84.

13. Whiley RA, Beighton D, Winstanley TG, Fraser HY, Hardie JM. Streptococcus intermedius, Streptococcus constellatus and Streptococcus anginosus (the Streptococcus milleri group): Association with Different Body Sites and Clinical Infections. J ClinMicrobiol 1992; 30: 243-4.

14. Wanahita A, Goldsmith EA, Musher DM, Clarridge JE 3rd, Rubio J, Krishnan B, et al. Interaction between human polymorphonuclear leukocytes and Streptococcus milleri group bacteria. J Infect Dis 2002; 185: 85-90.

15. Nagamune H, Ohnishi C, Katsuura A, Fushitani K, Whiley RA, Tsuji A, et al. Intermedilysin, a novel cytotoxin specific for human cells secreted by Streptococcus intermedius UNS46 isolated from a human liver abscess. Infect Immun 1996; 64: 3093-100.

16. Nagamune H, Whiley RA, Goto T, Inai Y, Maeda T, Hardie JM, et al. Distribution of the intermedilysin gene among the anginosus group streptococci and correlation between intermedilysin production and deep-seated infection with Streptococcus intermedius. J ClinMicrobiol 2000; 38: 220-6.

17. Ahmed NA, Petersen FC, Scheie AA. AI-2 quorum sensing affects antibiotic susceptibility in Streptococcus anginosus. J Antimicrob Chemother 2007; 60: 49-53.

18. Ahmed NA, Petersen FC, Scheie AA. AI-2/LuxS is involved in increased biofilm formation by Streptococcus intermedius in the presence of antibiotics. Antimicrob Agents Chemother 2009; 53: 4258-63.

19. Pecharki D, Petersen FC, Scheie AA. LuxS and expression of virulence factors in Streptococcus intermedius. Oral MicrobiolImmunol 2008; 23: 79-83.

20. Woo PC, Tse H, Chan KM, Lau SK, Fung AM, Yip KT, et al. Streptococcus milleri endocarditis caused by Streptococcus anginosus. DiagnMicrobiol Infect Dis 2004; 48: 81-8.

21. Petti CA. Detection and identification of microorganisms by gene amplification and sequencing. Clin Infect Dis 2007; 44: 1108-14.

22. Gómez-Garcés JL, Alós JI, Cogollos R. Bacteriologic characteristics and antimicrobial susceptibility of 70 clinically significant isolates of Streptococcus milleri group. DiagnMicrobiol Infect Dis 1994; 19: 69-73.

23. Jacobs JA, Stobberingh EE. In-vitro antimicrobial susceptibility of the Streptococcus milleri group (Streptococcus anginosus, Streptococcus constellatus and Streptococcus intermedius). J AntimicrobChemother, 1996; 37: 371-5.

24. Bantar C, Fernández Canigia L, Relloso S, Lanza A, Bianchini H, Smayevsky J. Species belonging to the Streptococcus milleri group: antimicrobial susceptibility and comparative prevalence in significant clinical specimens. J ClinMicrobiol 1996; 34: 2020-2.

25. Limia A, Jiménez ML, Alarcón T, López-Brea M. Five year analysis of antimicrobial susceptibility of the Streptococcus milleri group. Eur J ClinMicrobiol Infect Dis 1999; 18: 440-44.

26. Tracy M, Wanahita A, Shuhatovich Y, Goldsmith EA, Clarridge JE 3rd, Musher DM. Antibiotic susceptibilitiesof genetically characterized Streptococcus milleri group strains. Antimicrob Agents Chemother 2001; 45: 1511-4. 